\title{
Complex bend. II. A new optics solution
}

\author{
G. Wang, ${ }^{1}$ T. Shaftan, ${ }^{1,{ }^{*}}$ V. Smaluk, ${ }^{1}$ Y. Hidaka, ${ }^{1}$ O. Chubar, ${ }^{1}$ T. Tanabe,${ }^{1}$ \\ J. Choi, ${ }^{1}$ S. Sharma, ${ }^{1}$ C. Spataro, ${ }^{1}$ and N. A. Mesentsev ${ }^{2}$ \\ ${ }^{1}$ NSLS-II, Brookhaven National Laboratory, Upton, New York 11973, USA \\ ${ }^{2}$ Budker Institute of Nuclear Physics, pr. Lavrentyeva 30, Novosibirsk 630090, Russia
}

(Received 30 March 2019; published 13 November 2019)

\begin{abstract}
In our previous publications, we introduced a concept of complex bend, which is a bending element consisting of dipole poles, interleaved with strong focusing and defocusing quadrupole poles. An electron ring built from such elements features low emittance while preserving substantial room for insertion devices and associated lattice elements. In this paper, we present two new optics solutions for the complex bend which offer to substantially reduce the device length by removing the dipole poles. In the first of the solutions, the bending is realized by shifting the quadrupole poles along the curved horizontal axis. For the second solution, we use permanent magnet quadrupole poles installed into a wide gap of the conventional electromagnet. In this case, the resulting bending field in the magnet gap is a superposition of the quadrupole field from the poles and the dipole field from the conventional magnet. We present an analysis of the particle motion and synchrotron radiation emitted in such fields, as well as an assessment of the ring linear lattice that is composed of complex bend elements.
\end{abstract}

DOI: $10.1103 /$ PhysRevAccelBeams.22.110703

\section{INTRODUCTION}

In Refs. [1,2], we reported the conceptual details of a complex bend element, which is based on a sequence of strong focusing poles of alternating polarity, interleaved with pure dipole poles, providing bending. Splitting a long dipole into a sequence of short focusing poles produces a strong local focusing of the beam envelope, as well as the lattice functions, and results in a substantially lower emittance of the ring lattice. Indeed, as the horizontal emittance depends on the number of discrete dipoles in the lattice as $N^{3}$, we write

$$
\varepsilon_{x}=F \frac{E^{2}}{J_{x} N_{d}^{3}} \stackrel{C B}{\Rightarrow} F \frac{E^{2}}{J_{x}\left[N_{d} N_{p}\right]^{3}},
$$

where $F$ is a function of the ring lattice, $E$ is the beam energy, $J_{x}$ is the horizontal partition number, $N_{d}$ is the number of dipoles, and $N_{p}$ is the number of poles per dipole in the ring. Therefore, by using the complex bend concept, we expect to gain a large factor in the brightness of a synchrotron light source, which is a function of the ring emittance.

\footnotetext{
*shaftan@bnl.gov
}

Published by the American Physical Society under the terms of the Creative Commons Attribution 4.0 International license. Further distribution of this work must maintain attribution to the author(s) and the published article's title, journal citation, and DOI.
Recently [3], we have considered a modification of the complex bend element, aiming to reduce its overall length, lower the quadrupole strength, and, therefore, free up more space in the storage ring lattice available for installing lattice magnets, diagnostics, and insertion devices. We realized this modification by removing dipole poles from the element and enabling the bending either by quadrupole poles, shifted transversely, or by an external field introduced by an electromagnet with the quadrupole poles fitted inside its gap.

With these solutions, we designed the complex bend element, which is about half as long as the previous version with the dipole poles [1] at a comparable or lower gradient (we constrained ourselves to stay at or below $250 \mathrm{~T} / \mathrm{m}$ and at the bore diameter of not less than $1 \mathrm{~cm}$ ). We analyzed fields of the shifted quadrupoles and particle dynamics through them, which is presented in Sec. II of this paper. In the following, we applied the developed solution to a model ring lattice, taking NSLS-II ring geometry as the reference. In the process of our analysis, we found that the ring optics can be transversely and longitudinally stable only if a specific condition on the relationship between the shifts of focusing and defocusing poles is satisfied. This condition is described in Sec. III of this paper. Section IV presents a lattice option for the NSLS-II upgrade. Section V describes a realistic model of a single cell constructed with two quadrupoles shifted off their reference trajectory and considerations for the building of a prototype. We also discuss here the solution of the quadrupole poles aligned with respect to the curved complex bend element axis under the influence of the external dipole field. Section VI is dedicated to 
TABLE I. Parameters used in the analysis of complex bend II in this paper.

\begin{tabular}{lcc}
\hline \hline$N_{\text {dip }}$ & Number of CBII elements in the ring & 60 \\
$N_{\text {pdip }}$ & Number of cells per CBII element & 5 \\
$N_{p}=N_{\text {pdip }} \cdot N_{\text {dip }}$ & Total number of cells in the ring & 300 \\
$N_{d}=2 \cdot N_{p}$ & Total number of poles in the ring & 600 \\
$\alpha_{d}=2 \pi / N_{p}$ & Angle per cell, mrad & 20.94 \\
$\alpha_{\text {pdip }}=\alpha_{d} \cdot N_{\text {pdip }}$ & Angle per CBII element, mrad & 105 \\
$L_{Q}$ & Quadrupole pole length, m & 0.28 \\
$D_{d}$ & Drift length, m & 0.03 \\
$L_{\text {cell }}=2 \cdot D_{d}+2 \cdot L_{Q}$ & Cell length, m & 0.62 \\
$L_{\text {dip }}=L_{\text {cell }} \cdot N_{\text {pdip }}$ & Complex bend element length, m & 3.1 \\
$E / \gamma$ & Beam energy, GeV/unitless & $3 / 5871$ \\
$B R$ & Magnetic rigidity, T $\cdot \mathrm{m}$ & 10.0 \\
$K_{1 F} / K_{1 D}$ & Scaled gradient in CBII quadrupoles, m -2 & $25 /-25$ \\
$K_{1 F, D} \cdot B R$ & Corresponding field gradient, T/m \\
$\alpha$ & Fraction of QF angle in the cell's angle & $250,-250$ \\
\hline \hline
\end{tabular}

calculations of synchrotron radiation from complex bend poles. Throughout this paper, the QF-D-B-D-QD-D-B-D ${ }^{1}$ version will be called complex bend $\mathrm{I}(\mathrm{CBI})$ [1], and the $\mathrm{QF}$ D-QD-D version, either with shifted quadrupoles or with these aligned under a superimposed dipole field, will be called complex bend II (CBII). A conceptual design of complex bend with the superimposed dipole component is presented in Sec. VII.

\section{GENERAL ANALYSIS OF BEAM TRANSPORT IN A SHIFTED QUADRUPOLE}

In this section, we study particle dynamics in CBII that consists of several pairs of quadrupoles, every pair representing a single cell from the sequence of repetitive cells, with the "square wave" field distribution along the $s$ axis. The bending angle is realized by shifting both quadrupoles off center relative to the beam orbit. A single cell of the CBII contains a focusing quadrupole, a drift, a defocusing quadrupole, and another drift. In our analysis, we will be using the following parameters (Table I), which are relevant to the existing layout of the NSLS-II storage ring.

The total angle per single cell is distributed between the two quadrupoles according to the parameter $0<\alpha<1$. We define the entrance angles as

$$
\Delta x p_{F}=\frac{\alpha_{d}}{2} \alpha, \quad \Delta x p_{D}=\frac{\alpha_{d}}{2}(1-\alpha),
$$

yielding the constraint $2 \cdot\left(\Delta x p_{F}+\Delta x p_{D}\right)=\alpha_{d}$. In Fig. 1, we illustrate the principle of three consecutive poles or 1.5 cells of the CBII featuring the geometry of the beam orbit that consists of a sequence of arcs with a variable radius of curvature separated by short drifts. Initial conditions are matched on the interfaces between the elements.

Below, we include expressions for principle coordinates, trajectories, curvatures, and fields along the trajectories. We are separately considering $\mathrm{QF}$ and $\mathrm{QD}$ quadrupole poles. We use the following definitions for shortening the expressions:

$$
\begin{aligned}
\mu_{0.5 Q F, D} & =0.5 \cdot \sqrt{K_{1 F, D}} \cdot L_{Q}, \quad \mu_{Q F, D}=\sqrt{K_{1 F, D}} \cdot L_{Q}, \quad \mu_{F, D}=\sqrt{K_{1 F, D}} \cdot s, \\
C_{0.5 Q} & =\cos \left(\mu_{0.5 Q F}\right), \quad S_{0.5 Q}=\sin \left(\mu_{0.5 Q F}\right), \quad C_{Q}=\cos \left(\mu_{Q F}\right), \quad S_{Q}=\sin \left(\mu_{Q F}\right), \\
C & =\cos \left(\mu_{F}\right), \quad S=\sin \left(\mu_{F}\right), \\
C h_{0.5 Q} & =\cosh \left(\mu_{0.5 Q D}\right), \quad S h_{0.5 Q}=\sinh \left(\mu_{0.5 Q D}\right), \quad C h_{Q}=\cosh \left(\mu_{Q D}\right), \quad S h_{Q}=\sinh \left(\mu_{Q D}\right), \\
C h & =\cosh \left(\mu_{D}\right), \quad S h=\sinh \left(\mu_{D}\right) .
\end{aligned}
$$

Using this notation, we write the principal coordinates as

$$
\begin{aligned}
\Delta x_{F} & =\Delta x p_{F} \frac{S_{Q}}{\sqrt{K_{1 F}}\left(1-C_{Q}\right)}, \quad \Delta x_{D}=\Delta x p_{D} \frac{S h_{Q}}{\sqrt{K_{1 D}}\left(1-C h_{Q}\right)}, \\
\Delta x_{m F} & =\frac{\Delta x_{F}}{C_{0.5 Q}}, \quad \Delta x_{m D}=\frac{\Delta x_{D}}{C h_{0.5 Q}} .
\end{aligned}
$$

${ }^{1} \mathrm{QF}$ stands for the focusing quadrupole pole, QD stands for the defocusing one, and D stands for a drift. 


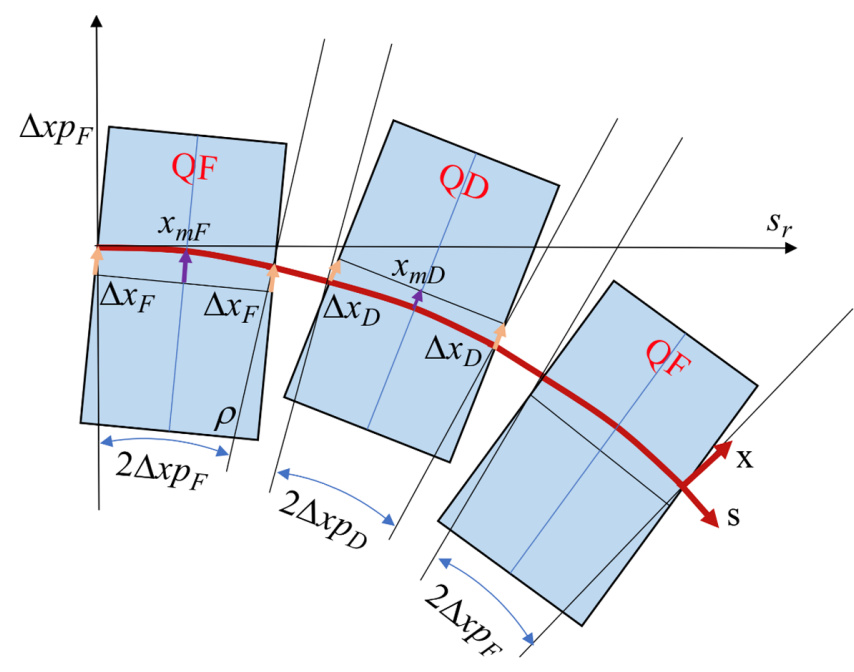

FIG. 1. Sketch of CBII geometry.

We assume that the trajectory comes in with an angle of $\Delta x p_{F, D}$ with respect to the pole face and leaves the pole with the same angle, so that the trajectory is symmetric relative to the midplane of the pole (Fig. 1). Then, for the coordinates of the beam trajectory, we get

$x_{F}=\Delta x_{F} C+\frac{\Delta x p_{F}}{\sqrt{K_{1 F}}} S, \quad x_{D}=\Delta x_{D} C h+\frac{\Delta x p_{D}}{\sqrt{K_{1 D}}} S h$,

where $C$ and $S$ are functions of $s$. We get the following expressions for the curvatures of the trajectories:

$$
\rho_{F, D}=\frac{1}{x_{F, D} \cdot K_{1 F, D}}, \quad \rho_{\mathrm{Fav}, \mathrm{Dav}}=\frac{1}{L_{Q}} \int_{0}^{L_{Q}} \rho_{F, D} d s,
$$

where $\rho_{\text {Fav }}$ and $\rho_{\text {Dav }}$ are the average trajectory radii.
The following expressions hold for the fields along these trajectories:

$$
B_{F, D}=\frac{\mathrm{BR}}{\rho_{F, D}(s)}, \quad B_{\mathrm{Fav}, \mathrm{Dav}}=\frac{1}{L_{Q}} \int_{0}^{L_{Q}} B_{F, D} d s,
$$

where $B_{\mathrm{Fav}}$ and $B_{\mathrm{Dav}}$ are average fields along the trajectory. We plot the trajectories, curvatures, and fields in Fig. 2.

To find the length of the trajectory through the pole, we write

$$
\begin{aligned}
L_{F, D} & =2 \int_{0}^{0.5 L_{Q}} \sqrt{1+\Delta x_{m F, D}^{2} \cdot K_{1 F, D} \cdot S^{2}} d s \\
& \approx L_{Q}+\frac{K_{1 F, D}{ }^{2} \cdot L_{Q}^{3} \cdot \Delta x_{m F, D}{ }^{2}}{24},
\end{aligned}
$$

where the last expression is obtained by expressing the square root in the Taylor series and retaining only the first term.

Next, we estimate the linear part of the edge focusing in the horizontal plane, since it may perturb the lattice functions affecting the horizontal emittance, which we intend to minimize. For the angle gained by the particle while passing the fringe field of the CBII pole, we write

$$
\Delta x^{\prime} \approx \frac{1}{\mathrm{BR}} \int_{0}^{\Delta x_{F} \Delta x p_{F}} B(s) d s \approx K_{1 F} \Delta x_{F}^{2} \Delta x p_{F},
$$

where we assumed that the field along the wedge covered by $\Delta x p_{F}$ is constant. In the "square wave" field model, the angle $\Delta x^{\prime}$ is much smaller $\left(\sim \Delta x^{3}\right)$ than that produced as a result of focusing by the field in the pole's body $(\sim \Delta x)$.

Then, we work out the matrices of the quadrupole poles shifted by $\Delta x_{F}$ and tilted by $\Delta x p_{F}$, assuming that the trajectory follows Eqs. (1). In this case, all terms but the dispersive ones will be the same as for the normal quadrupole.
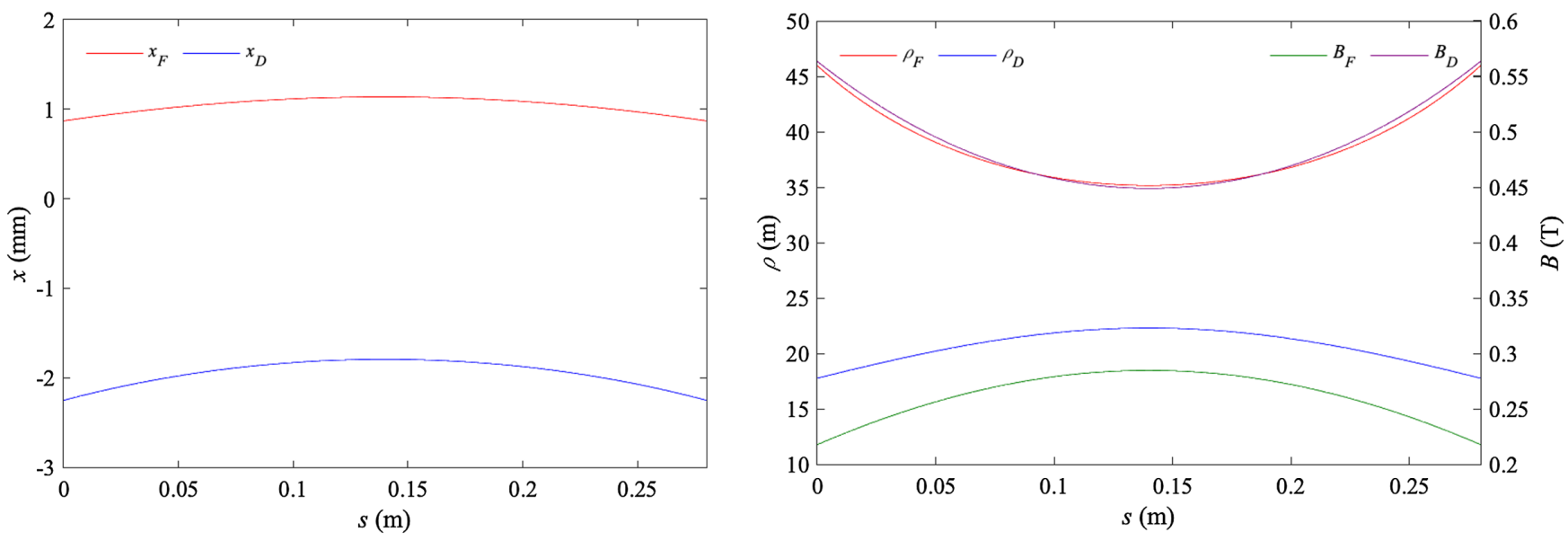

FIG. 2. Trajectories, their curvatures, and fields along a single pole with length $L_{Q}$. 
First, we derive it by referencing the trajectory with respect to Eqs. (1), substituting $K_{1} \rightarrow \sqrt{\frac{K_{1}}{1+\delta}}$, expanding the resulting expression in $\delta$, and keeping only the terms linear in $\delta$ :

$$
\begin{aligned}
x(s) \approx & x_{o} C+\frac{x_{o}^{\prime} S}{\sqrt{K_{1 F}}}+\frac{\delta}{2}\left(-\Delta x p_{F}\left(L_{Q} C-\frac{S}{\sqrt{K_{1 F}}}\right)\right. \\
& \left.+\Delta x_{F} \sqrt{K_{1 F}} L_{Q} S\right)
\end{aligned}
$$

where $x_{o}$ and $x_{o}^{\prime}$ are deviations from the reference (1).

We cross-check this expression by directly solving the equation of motion through, for example, a $\mathrm{QF}$ pole:

$$
x^{\prime \prime}+K_{1 F} x=\frac{\delta}{\rho(s)},
$$

where

$$
\frac{1}{\rho(s)}=\frac{B(s)}{\mathrm{BR}}=K_{1 F}\left[\Delta x_{F} C+\frac{\Delta x p_{F}}{\sqrt{K_{1 F}}} S\right]
$$

Integrating this equation analytically, we get the same expression for the dispersive term as in Eq. (2):

$$
x(s)=-\frac{\Delta x p_{F}}{2}\left(L_{Q} C-\frac{S}{\sqrt{K_{1 F}}}\right)+\frac{\Delta x_{F}}{2} \sqrt{K_{1 F}} L_{Q} S .
$$

As a result of a similar analysis, we get the following matrices for the CBII poles in variables $\left(x, x^{\prime}, \delta\right)^{T}$ :

$$
\begin{gathered}
M F=\left|\begin{array}{ccc}
C_{Q} & S_{Q} / \sqrt{K_{1 F}} & -\frac{\Delta x p_{F}}{2}\left(L_{Q} C_{Q}-\frac{S_{Q}}{\sqrt{K_{1 F}}}\right)+\frac{\Delta x_{F}}{2} \sqrt{K_{1 F}} L_{Q} S_{Q} \\
-S_{Q} \sqrt{K_{1 F}} & C_{Q} & \frac{\Delta x_{F}}{2}\left(\sqrt{K_{1 F}} S_{Q}+K_{1 F} L_{Q} C_{Q}\right)+\frac{\Delta x p_{F}}{2} \sqrt{K_{1 F}} L_{Q} S_{Q} \\
0 & 0 & 1
\end{array}\right|, \\
M D=\left|\begin{array}{ccc}
C h_{Q} & S h_{Q} / \sqrt{K_{1 D}} & \frac{\Delta x p_{D}}{2}\left(-L_{Q} C h_{Q}+\frac{S h_{Q}}{\sqrt{K_{1 D}}}\right)-\frac{\Delta x_{D}}{2} \sqrt{K_{1 D}} L_{Q} S h_{Q} \\
S h_{Q \sqrt{K_{1 D}}} & C h_{Q} & -\frac{\Delta x_{D}}{2}\left(\sqrt{K_{1 D}} S h_{Q}+K_{1 D} L_{Q} C h_{Q}\right)-\frac{\Delta x p_{D}}{2} \sqrt{K_{1 D}} L_{Q} S_{h Q} \\
0 & 0 & 1
\end{array}\right|,
\end{gathered}
$$

where $M_{13}$ and $M_{23}$ are different from those in the transport matrix for the combined function magnet with the equivalent $\rho=\rho_{\text {Fav,Dav }}$ and $K_{1 F, D}$ strengths.

For such a magnet, we get $M_{13}$ and $M_{23}$ as

$$
M F_{13}=\frac{1}{\rho_{\mathrm{Fav}} K_{1 F}}(1-C), \quad M F_{23}=\frac{1}{\rho_{\text {Fav }} K_{1 F}} S
$$

for the focusing combined function magnet and similar expressions for the defocusing combined function magnet:

$$
M D_{13}=\frac{-1}{\rho_{\mathrm{Dav}} K_{1 D}}(1-C h), \quad M D_{23}=\frac{1}{\rho_{\mathrm{Dav}} K_{1 D}} S h .
$$

Next, we describe a single cell of CBII in two ways: $M F_{0.5} M_{d} M D M_{d} M F_{0.5}$ and $M D_{0.5} M_{d} M F M_{d} M D_{0.5}$, where the resulting matrices correspond to the maxima and minima of the beam envelopes through the element. Using these matrices, we find stable solutions in terms of $\mu_{x, y}, \bar{\beta}_{x, y}, \bar{\eta}$ and $\Delta \beta_{x, y}, \Delta \eta$.

Then we approximate the beta function and dispersion through the cell using expressions like

$$
\left[\begin{array}{c}
\beta_{x}(s) \\
\eta(s)
\end{array}\right] \approx\left[\begin{array}{c}
\bar{\beta}_{x} \\
\bar{\eta}
\end{array}\right]+\left[\begin{array}{c}
\Delta \beta_{x} \\
\Delta \eta
\end{array}\right] \cdot \cos \left(k_{C B} s\right)
$$

with $k_{C B}=\frac{2 \pi}{L_{\text {cell }}}$, and $\Delta \beta, \Delta \eta$ corresponds to the difference between $\beta, \eta$ in each pole. These solutions are plotted in Fig. 3.

With these approximate solutions, we find expressions for the storage ring emittance and energy spread.

The average dispersion and beta function in each quad are, respectively,

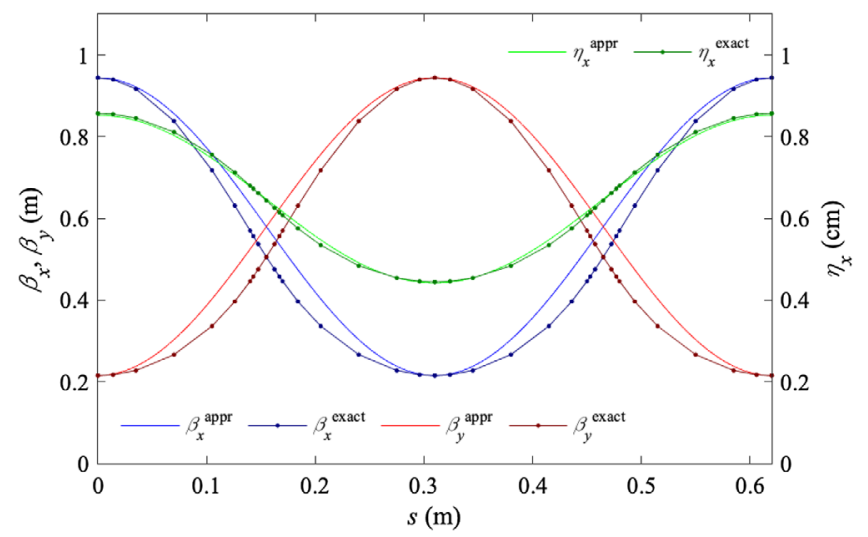

FIG. 3. Approximate solutions (solid lines) for horizontal and vertical beta function and dispersion as compared with their exact solutions (squares and thin lines) within a single CBII cell. 


$$
\begin{aligned}
& \left(\begin{array}{c}
\eta_{\mathrm{Fav}} \\
\beta_{\mathrm{Fav}}
\end{array}\right)=\frac{2}{L_{Q}} \int_{0}^{L_{Q} / 2}\left(\begin{array}{c}
\eta(s) \\
\beta(s)
\end{array}\right) d s, \\
& \left(\begin{array}{c}
\eta_{\mathrm{Dav}} \\
\beta_{\mathrm{Dav}}
\end{array}\right)=\frac{2}{L_{Q}} \int_{\left(L_{Q} / 2\right)+D_{d}}^{L_{Q}+D_{d}}\left(\begin{array}{c}
\eta(s) \\
\beta(s)
\end{array}\right) d s .
\end{aligned}
$$

The contribution from the CBII elements to the tune and chromaticity of the whole ring are

$$
\begin{aligned}
\nu_{x, y}= & N_{p} \cdot \mu_{x, y}, \\
\xi_{x, y} \approx & -\frac{N_{p}}{4 \pi}\left(2\left(K_{1 F}-K_{1 D}\right) \frac{\Delta \beta_{x, y}}{k_{C B}} \sin \left(\frac{k_{C B} L_{Q}}{2}\right)\right. \\
& \left. \pm\left(K_{1 F}+K_{1 D}\right) L_{Q} \bar{\beta}_{x, y}\right)
\end{aligned}
$$

with $\mu_{x, y}$ being the phase advance per cell. $I_{2}, I_{4}$, and $I_{5}$ are the radiation integrals around the ring. The radiation integrals for the whole set of the CBII elements can be expressed as

$$
\begin{aligned}
& I_{2}=2 \pi\left(\frac{\alpha}{\rho_{\mathrm{Fav}}}+\frac{(1-\alpha)}{\rho_{\mathrm{Dav}}}\right), \\
& I_{3}=2 \pi\left(\frac{\alpha}{\rho_{\mathrm{Fav}}^{2}}+\frac{(1-\alpha)}{\rho_{\mathrm{Dav}}^{2}}\right), \\
& I_{4} \approx \frac{N_{p} \cdot 2 \eta_{\mathrm{Fav}} \cdot K_{1 F} \cdot L_{Q}}{\rho_{\mathrm{Fav}}}-\frac{N_{p} \cdot 2 \eta_{\mathrm{Dav}} \cdot K_{1 D} \cdot L_{Q}}{\rho_{\mathrm{Dav}}},
\end{aligned}
$$

where we neglected the integral contributions from $\frac{\eta}{\rho^{3}}$, since they are much smaller ${ }^{2}$ than those terms containing $K_{1}$, and

$$
I_{5} \approx 2 \pi\left(\frac{\alpha}{\rho_{\text {Fav }}^{2}} \frac{\eta_{\text {Fav }}^{2}}{\beta_{\text {Fav }}}+\frac{(1-\alpha)}{\rho_{\text {Dav }}^{2}} \frac{\eta_{\text {Dav }}^{2}}{\beta_{\text {Dav }}}\right) .
$$

The storage ring emittance and energy spread are [5], respectively,

$$
\begin{aligned}
\varepsilon_{x} & =C_{q} \gamma^{2} \frac{I_{5}}{I_{2}-I_{4}}=C_{q} \cdot \gamma^{2} \cdot \frac{I_{5}}{J_{x} \cdot I_{2}}, \\
\frac{\sigma_{E}}{E} & =\gamma \sqrt{C_{q} \cdot \frac{I_{3}}{2 I_{2}+I_{4}}}, \\
\frac{\sigma_{E}}{E} & =\gamma \sqrt{C_{q} \cdot \frac{I_{3}}{2 I_{2}+I_{4}}},
\end{aligned}
$$

where $C_{q} \approx 3.84 \times 10^{-13} \mathrm{~m}$.

For the momentum compaction, we write (the approximation is valid when $L_{Q} \gg D_{d}$ )

\footnotetext{
${ }^{2}$ This term in $I_{4}$ is equal to zero for a rectangular dipole magnet [4].
}

$$
\begin{aligned}
\alpha_{\mathrm{MC}}= & \frac{1}{C} \oint \frac{\eta}{\rho} d s \approx \frac{1}{2 L_{Q}}\left(2 \int_{0}^{L_{Q} / 2} K_{1 F} \cdot x_{F} \cdot \eta \cdot d s\right. \\
& \left.+\int_{\left(L_{Q} / 2\right)+D_{d}}^{\left(3 L_{Q} / 2\right)+D_{d}} K_{1 D} \cdot x_{D} \cdot \eta \cdot d s\right),
\end{aligned}
$$

where $C$ is the circumference, $K_{1 F, D} \cdot x_{F, D}=\frac{1}{\rho_{F, D}}$, and $\eta(s)=\bar{\eta}+\Delta \eta \cdot \cos \left(k_{C B} s\right)$, as we have shown in this section.

With these expressions in mind, we are in the position to describe the storage ring lattice along the arcs. All the ring arcs consist of CBII elements, which define the ring optics in terms of emittance, energy spread, and momentum compaction, and largely affect tunes, chromaticity, driving terms, and dynamic and momentum apertures.

Before computing these ring parameters, we need to derive an important dependence for the relationship between focusing and defocusing poles in a CBII element. This dependence defines the stability of the ring built with CBII elements.

\section{STABILITY CONSTRAINT FOR THE RING OPTICS BASED ON COMPLEX BEND II}

As per expressions from Sec. II, $J_{x}$ is determined by $I_{2}$ and $I_{4}$, and, in turn, $I_{2}$ depends only on the dipole bending radius. To maintain the longitudinal or horizontal stability of the beam dynamics in the ring, we need to ensure that $J$ elements of the damping distribution are always positive. The complex bend optics allow us to simplify the synchrotron integrals presented above [1]. Following Eq. (5), for a sequence of $N_{F}$ focusing and $N_{D}$ defocusing shifted quadrupole magnets, the fourth synchrotron integral $I_{4}$ can be approximated as

$$
I_{4} \approx \int \frac{2 \eta_{F} K_{1 F}}{\rho_{F}} d s+\int \frac{2 \eta_{D} K_{1 D}}{\rho_{D}} d s .
$$

The change in $I_{4}$ due to CBII poles or combined function magnets can be estimated as

$$
I_{4} \approx 2 N_{F} L_{F} \eta_{F a v} K_{1 F}^{2} \Delta x_{F}+2 N_{D} L_{D} \eta_{\text {Dav }} K_{1 D}^{2} \Delta x_{D} .
$$

For a periodic structure, $N_{F}=N_{D}=N_{Q}, L_{F}=L_{D}=L_{Q}$. In the particular case when there is no net change in $I_{4}$ due to CBII poles or combined function magnets, we determine that the relationship between the translations and gradients of the focusing and defocusing poles is

$$
\eta_{\text {Fav }} K_{1 F}^{2} \Delta x_{F}+\eta_{\text {Dav }} K_{1 D}^{2} \Delta x_{D}=0
$$

This constraint maintains the ring optics with complex bends II stable in all three planes by preserving damping partitions positive. We note that this constraint works for any ring optics, combined function magnets, or shifted quadrupoles, i.e., CBII elements. In the actual lattice design, one may adjust this constraint to reach minimum 
TABLE II. Preliminary parameters of a storage ring based on complex bend II. Horizontal emittance is 35 pm-rad at $3 \mathrm{GeV}$.

\begin{tabular}{lc}
\hline \hline Circumference, m & 792 \\
Emittance, pm-rad & 34.5 \\
Energy spread, \% & 0.07 \\
Momentum compaction & $3.93 \times 10^{-5}$ \\
Natural chromaticity & $-229 /-255$ \\
Tunes & $96.12 / 119.86$ \\
Beta functions in straights, m & $3.3 / 1.8$ \\
Ratio of straights to circumference & $28.6 \%$ \\
Synchrotron radiation power, keV/turn & 309 \\
\hline \hline
\end{tabular}

emittance by increasing $J_{x}$, which should maintain damping partitions always positive.

\section{PARAMETERS OF COMPLEX BEND II FOR AN OPTION OF THE NSLS-II UPGRADE}

Using the formalism described in Secs. II and III, we designed a storage ring lattice with complex bend II elements in the place of the conventional dipoles of double bend achromat lattice. Using the CBII transport matrices, we calculated matched solutions for Twiss functions and dispersion. Then we estimated the ring parameters and optimized them, yielding the stability constraint described in Sec. III.

In our design, we used the parameters of CBII element from Table I. The shifts of QF and QD poles are 1.13 and $-1.79 \mathrm{~mm}$, correspondingly. The latter shift is large as compared with the total diameter of the aperture inside the poles of $10 \mathrm{~mm}$, and we assessed a solution for the magnet design, which enables us to reduce this shift.

In Table II, we presented the ring parameters using the expressions from Sec. II and confirmed by a lattice code.

The lattice functions are presented in Fig. 4.

The arrangement of the quadrupoles looks similar between the two lattices; however, the CBII lattice features an emittance that is a factor of 70 smaller than that for the NSLS-II DBA lattice. We showed only chromatic sextupole

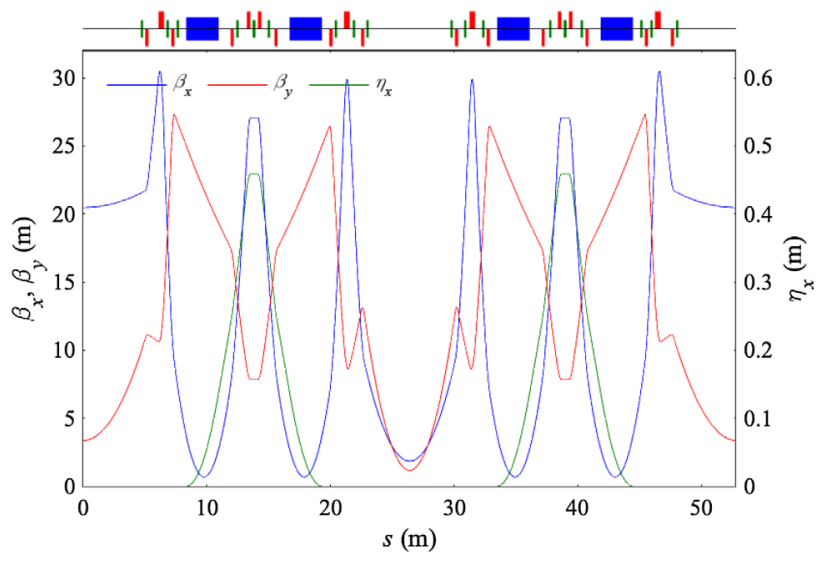

magnets on the right plot, while the harmonic sextupoles' location $\left(K_{2} L<120 \mathrm{~m}^{-1}\right)$ and strength are being optimized. Other optimization constraints include the chromaticity and dispersion wave between the CBII elements.

\section{MODEL OF THE COMPLEX BEND II WITH SHIFTED QUADRUPOLES}

In this section, we analyzed a model of the quadrupole with straight edges and constant gradient, shifted by $1 \mathrm{~mm}$ in the horizontal direction. We compared transport through this magnetic element using several different models in Elegant [6], including KQUAD (particle tracked using symplectic drift-kick integration through a canonical quadrupole element), BMAPXY (particle tracked using the Runge-Kutta integration method through a model of a transverse magnetic field), and BMXYZ (particle tracked using the Runge-Kutta integration method through a model of a 3D magnetic field specified as a field map) with the analytical solution (3).

The comparison is presented in Fig. 5 below. We used the $3 \mathrm{GeV}$ parameters from Table II, and a nominal drift space of $0.03 \mathrm{~m}$ is assumed.

The trajectories calculated with Elegant agree with the theoretical estimates to stay within $10 \mathrm{~nm}$ (Fig. 5).

As follows from the study above, the first-order estimate (4) describes the particle motion through the shifted quadrupole with sufficient accuracy to calculate of the horizontal beta function and linear dispersion and, in turn, to calculate the emittance. We are aware that shifting QF and QD for generating the bending field will generate higher harmonics of the field distribution within the gap. These harmonics can be compensated either by chamfering of the poles or by correction coils.

In Table III, we also presented the parameters of the complex bend prototype for future experiments at Brookhaven Lab's Accelerator Test Facility (ATF), evaluated at a beam energy of $50 \mathrm{MeV}$. The CBII prototype will be $46 \mathrm{~mm}$ long and consists of five cells, with the field gradient set at $150 \mathrm{~T} / \mathrm{m}$. We scaled the complex bend parameters from

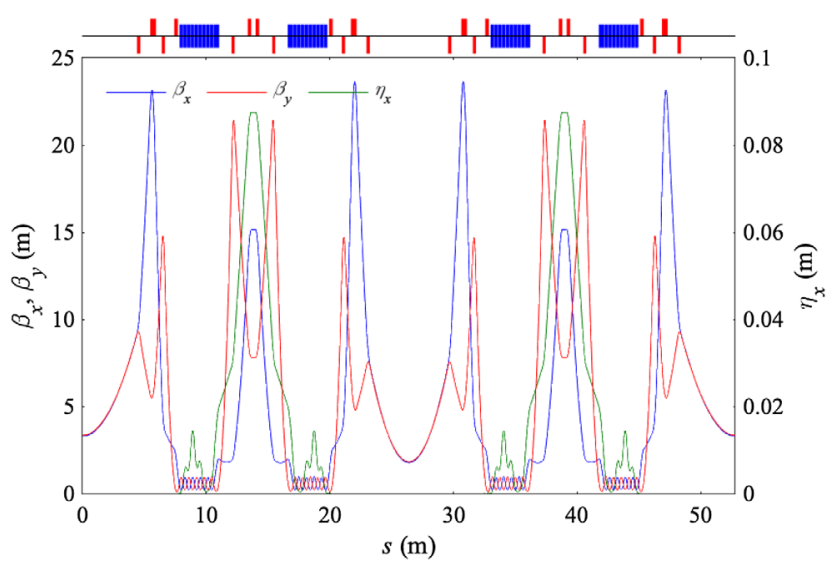

FIG. 4. Comparison of the two lattices: NSLS-II DBA and NSLS-II upgrade based on the CBII design. 


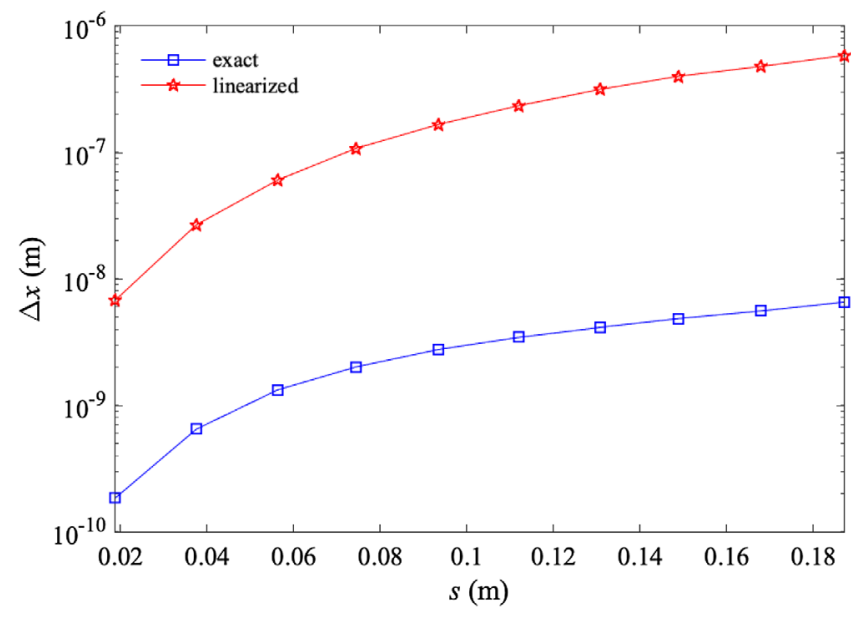

FIG. 5. The difference between the results, obtained with the exact solution of (3) (blue squares), and the linearized solution (2) (red stars) relative to tracking through magnetic fields specified by the BMXYZ element in the Elegant model.

$3 \mathrm{GeV}$ down to $50 \mathrm{MeV}$, which corresponds to a reduction in magnetic rigidity $(B R)$ by a factor of $C_{E}=0.017$. We also reduced the quadrupole pole length by a factor of $C_{L}=6$ while keeping the values of the bend angle and $\sqrt{K_{1}} L_{Q}$ the same as for the $3 \mathrm{GeV} \mathrm{CBII} \mathrm{cell.} \mathrm{The} \mathrm{drift} \mathrm{between} \mathrm{the} \mathrm{poles}$ is reduced by a factor of 2, taking into account the space limitations. Thus, overall, the cell length is scaled down by a factor of $C_{L}$ and becomes $12.3 \mathrm{~cm}$. The scaling of the dipole field follows $B L / B R$; therefore, the dipole component induced by the quadrupole poles along the beam orbit is reduced by a factor of $C_{E} * C_{L} \sim 0.1$ as compared with the value at $3 \mathrm{GeV}$. The quadrupole $K_{1}$ and $L_{Q}$ change to maintain $\sqrt{K_{1}} L_{Q}$ constant, thereby increasing $K_{1}$ by a factor of $C_{L}^{2}=36$.

\section{CALCULATIONS OF SYNCHROTRON RADIATION FROM A COMPLEX BEND}

Here we briefly describe properties of synchrotron radiation (SR) from the $\mathrm{CB}$ element as it was installed in a $3 \mathrm{GeV}$ ring. The emission simulations were performed with the "Synchrotron Radiation Workshop" (SRW) computer code [7], using its new Python interface [8].
The central orbit and magnetic field "seen" by an electron moving along this orbit, for the complex bend parameters from Table I, are shown in Fig. 6. This calculation was performed using the 3D Runge-Kutta solver available in SRW, with a modeling magnetic field of quadrupoles with "soft" edges, at their nominal positions and orientations in the laboratory frame.

Figure 7 presents a calculation power density distribution of the synchrotron radiation (integrated over all photon energies) from the central portion of the complex bend model shown in Fig. 6. The calculations were performed for a transverse plane at $10 \mathrm{~m}$ observation distance from the center of the modeling magnetic field definition (point $\mathrm{ct}=0$; see Fig. 6).

The calculated radiation pattern consists of "lobes" corresponding to intensity peaks from individual poles in the complex bend structure, separated by "dips" (power density minima) along directions defined by the axes of straight intervals between the neighboring poles. Because of the relatively small observation distance (compared to the field definition range), the calculated distributions are impacted by the geometrical "near field" effect, resulting in a gradient of the power density vs the horizontal position in the observation plane. The positive horizontal position in these calculations corresponds to the direction towards outside of the storage ring. Note that since the magnetic field seen by electrons in the quads is not constant along their trajectories, the power density generated in each quad is also modulated along the horizontal position and angle (decreasing towards angles corresponding to edges of the defocusing quad and increasing towards the angle corresponding to edges of the focusing quads). However, these effects are not expected to represent significant difficulties for using this radiation in x-ray beam lines (that typically have acceptance angles not exceeding several milliradians).

We also calculated the spectra of SR intensity (spectral flux per unit surface area) at different horizontal positions at a zero vertical position in the same observation plane (located at $10 \mathrm{~m}$ from the center of the magnetic field definition interval) as shown in Fig. 8.

The spectrum at $x=0$ corresponds to the emission point in the middle of a defocusing quad. The other spectral

TABLE III. Parameters of NSLS-II dipole, complex bend used in the calculation, and a prototype for a future CBII optics test at BNL's ATF.

\begin{tabular}{lccc}
\hline \hline & NSLS-II dipole & Complex bend & 50 MeV prototype \\
\hline Length, m & 2.6 & 3.1 & 0.62 \\
Bending field, T & 0.4 & $0.26 / 0.49$ & $0.026 / 0.049$ \\
Cell length, cm & $\ldots$ & 62 & 12.3 \\
Bending angle per cell, ${ }^{\circ}$ & 6 & 1.2 & 1.2 \\
Gradient, T/m & 0 & $250 /-250$ & $150 /-150$ \\
$\beta_{X \max } / \beta_{X \min }, \mathrm{m}$ & $3.7 / 0.7$ & $0.94 / 0.22$ & $0.26 / 0.018$ \\
$\eta_{\max } / \eta_{\min }, \mathrm{mm}$ & $137 / 0$ & $4.41 / 8.52$ & $1 . / 0.4$ \\
\hline \hline
\end{tabular}



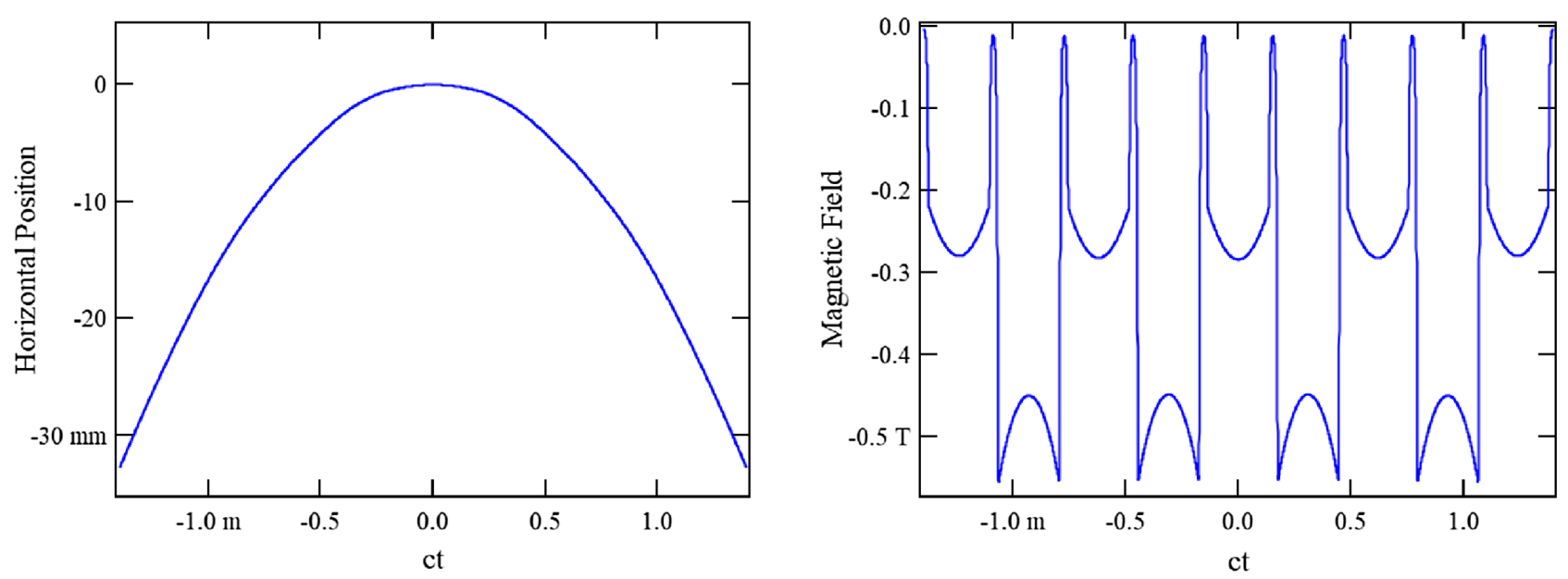

FIG. 6. Central trajectory in millimeters through five poles of the CB element and magnetic field in Tesla seen by an electron moving along this trajectory (plotted in meters). The first pole of CBII in this calculation is taken as a vertically focusing element.
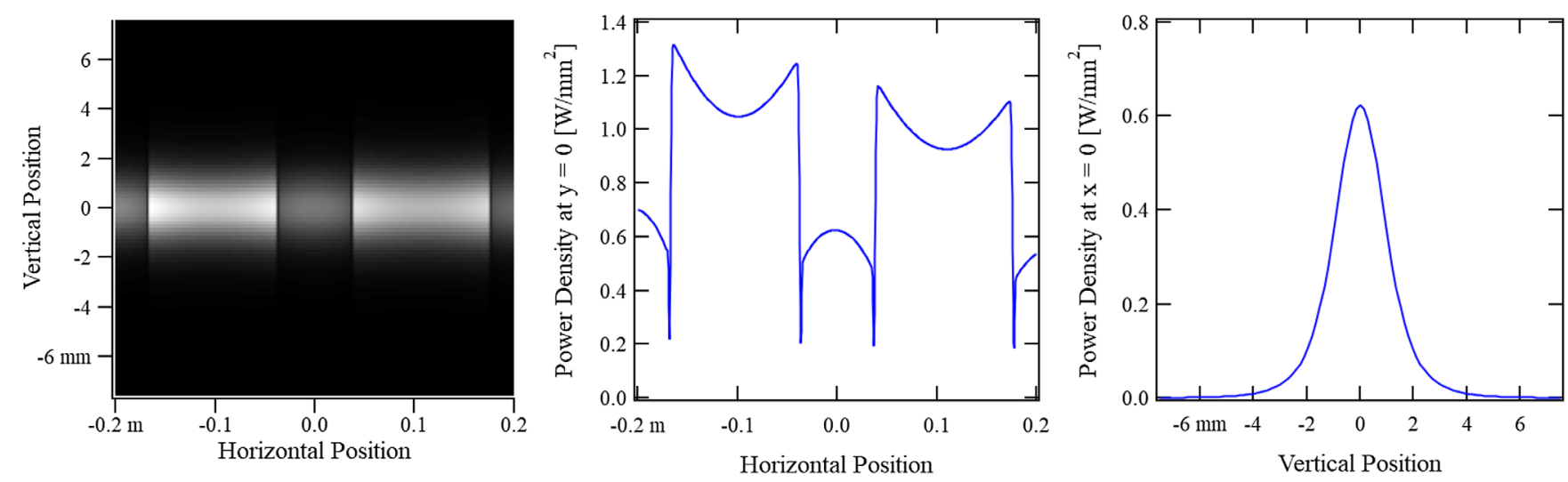

FIG. 7. SR power density distributions from the complex bend calculated in the transverse plane located at $10 \mathrm{~m}$ away from the center of the magnetic field regions.

curves at $x=-0.05 \mathrm{~m}$ and $x=-0.1 \mathrm{~m}$ correspond, respectively, to the emission points near the edge and in the middle of a focusing quad.

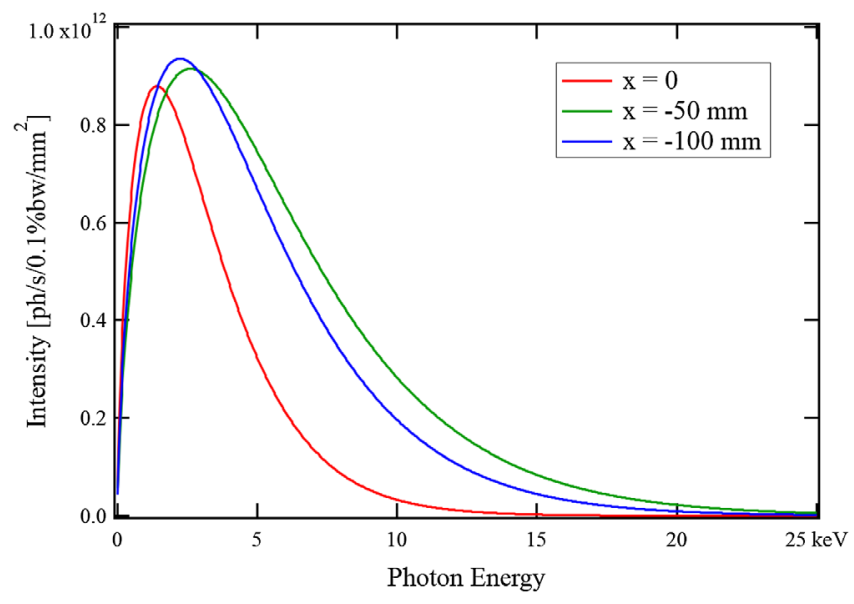

FIG. 8. SR intensity spectra corresponding to different emission points in defocusing and focusing quads of the complex bend II.

\section{CONCEPTUAL DESIGN OF COMPLEX BEND II COMPONENTS}

The quadrupole gradient is reduced to $\sim 250 \mathrm{~T} / \mathrm{m}$ in complex bend II as compared to $\sim 450 \mathrm{~T} / \mathrm{m}$ in complex bend I. Even the reduced gradient is still quite high but is

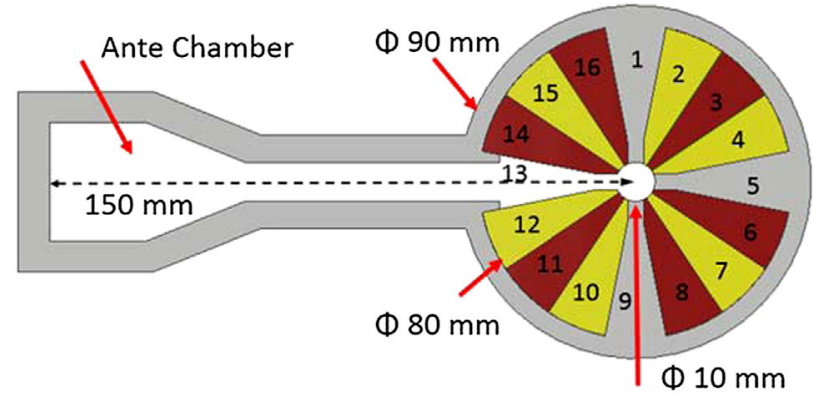

FIG. 9. Conceptual design of a high-gradient Halbach PMQ assembled inside a vacuum chamber. The PM wedge 13 is removed for the x-ray exit slot, and wedges 1, 5, and 9 are replaced with aluminum wedges to maintain fourfold symmetry. 
TABLE IV. Field harmonics of a modified Halbach PMQ with a minimum exit aperture of $4 \mathrm{~mm}$.

\begin{tabular}{lrcrrrrrr}
\hline \hline$n$ & 1 & 2 & 3 & 4 & 5 & 6 & 7 & 8 \\
\hline$A_{n}$ & 1.0 & 1.2 & 0.4 & 0.2 & 0.1 & 0.0 & 0.0 & 0.0 \\
$B_{n}$ & -1.5 & $10^{4}$ & 0.1 & -0.1 & -0.1 & -75.1 & 0.0 & 0.0 \\
\hline \hline
\end{tabular}

achievable with a small beam aperture of $\sim 10 \mathrm{~mm}$. Three conceptual designs of high-gradient quadrupoles with a $10 \mathrm{~mm}$ aperture are briefly presented in Ref. [9]. Here we describe in some detail one of these designs, namely, a 16wedge Halbach permanent magnet quadrupole (PMQ), a prototype of which we are presently building.

Figure 1 show the cross section of the Halbach PMQ assembled inside a round aluminum vacuum chamber of $90 \mathrm{~mm}$ diameter. The inside and outside diameters of the PM wedges are 10 and $80 \mathrm{~mm}$, respectively. The vacuum chamber has a bend radius of $\sim 25 \mathrm{~m}$ and length of a cell of complex bend $(\sim 280 \mathrm{~mm})$. On the outboard side, a typical antechamber of $150 \mathrm{~mm}$ width is provided for the extraction of $\mathrm{x}$ rays and for pumping via non evaporable getters (NEG) strips. This configuration requires removing wedge 13 of the standard Halbach PMQ and minor machining of wedges 12 and 14 for a minimum exit aperture of $4 \mathrm{~mm}$. This is repeated at the left, top, and bottom to maintain a fourfold symmetry for minimizing field harmonics. Removed PM wedges 1, 5, and 9 are replaced by aluminum wedges to provide structural restraint to the other PM wedges.

A 3D Opera model of the PMQ (Fig. 9) with NdFeB PM wedges (remnant field of $1.12 \mathrm{~T}$ ) yields a quadrupole gradient of $237 \mathrm{~T} / \mathrm{m}$. Field harmonics calculated at $r=$ $2 \mathrm{~mm}$ per $10^{4}$ units of $B_{2}$ are shown in Table IV. All harmonics are acceptable except B6 (-75.1 units), which can be reduced by shimming or by adjusting the magnetization angles of the PM wedges.

The required dipole field for CBII can be provided conveniently by offsetting the Halbach PMQs in the

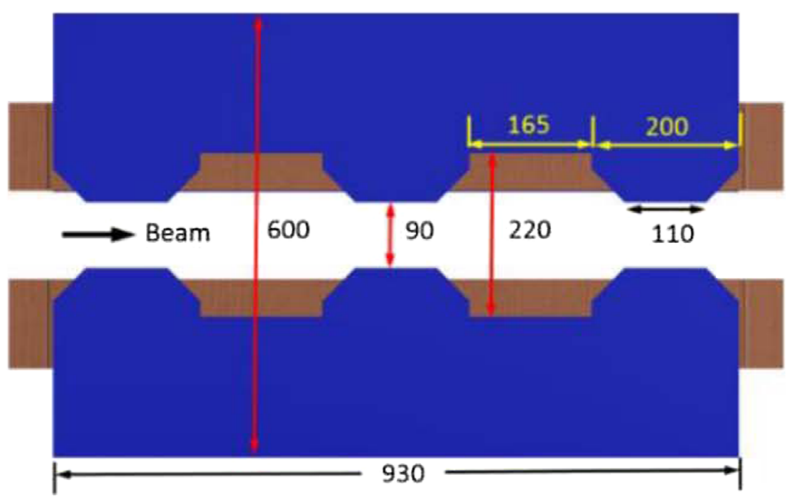

(a) transverse $x$ direction. However, the offset required for a dipole field $0.49 \mathrm{~T}$ is $\sim 2 \mathrm{~mm}$, which results, if not corrected, in large $B_{3}$ to $B_{6}$ (in the hundred unit range). Alternatively, the required field can be generated by an $\mathrm{H}$-shaped electromagnet with a $90 \mathrm{~mm}$ pole gap, in which the vacuum chamber-PMQ assemblies (Fig. 10) will be placed. The external dipole field can be superimposed [10] on Halbach PMQs because of full saturation $(\mu \approx 1.0)$ of the permanent magnets. With a pole width of $250 \mathrm{~mm}$ and coil current of $18000 \mathrm{~A}$ (current density of $4 \mathrm{~A} / \mathrm{mm}^{2}$ ), the dipole can generate a field of $0.49 \mathrm{~T}$ in arclengths occupied by defocusing PMQs. To reduce this field to $0.26 \mathrm{~T}$ in arclengths occupied by focusing PMQs, the pole gap is increased to $220 \mathrm{~mm}$.

A truncated complex bend II consisting of three small apertures of $90 \mathrm{~mm}$ and two large apertures of $220 \mathrm{~mm}$ [Fig. 10(a)] was modeled by Opera 3D. The chamfered sawtooth poles, $110 \mathrm{~mm}$ at the tip and $200 \mathrm{~mm}$ at the base, are placed $165 \mathrm{~mm}$ apart. The $\mathrm{H}$-shaped soft-iron (AISI 1006) magnet is $700 \mathrm{~mm}$ in width and contains two coils of $48 \mathrm{~mm} \times 96 \mathrm{~mm}$ in cross section. This dipole generates the $0.5 \mathrm{~T}$ dipole field with maximum and minimum field values in the required range [Fig. 10(b)].

\section{CONCLUSION}

In this paper, we presented an analysis of the complex bend II geometry based on the quadrupole poles with alternating polarity, shifted in the horizontal direction to acquire the required bending. First, we analyzed beam dynamics in the CBII element and then worked out NSLSII upgrade lattice solutions, where the original NSLS-II dipoles were replaced with the elements of the complex bend II. We presented a constraint that keeps the ring optics stable if the latter is based on combined function magnets or shifted quadrupoles corresponding to the CBII poles.

Next, we calculated all basic parameters of the ring based on the CBII concept and confirmed them with Elegant calculations. We reported in this paper results of our

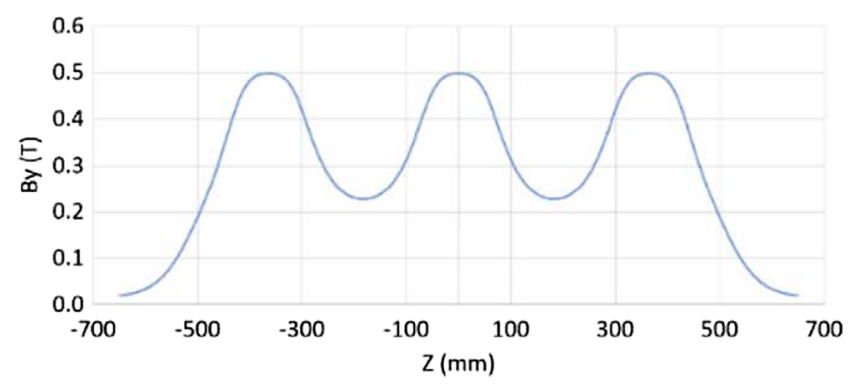

FIG. 10. External dipole for complex bend II, (a) variable aperture along the beam direction, and (b) dipole field along the beam direction. 
simulations of synchrotron radiation from the complex bend structure.

One of the clear advantages of complex bend II, as compared to complex bend $\mathrm{I}$, is a reduction of the overall length of the element structure due to the absence of the dipole poles. Overall, this approach reduces the design complexity of a single cell, as well as helps to avoid very high gradients at $500 \mathrm{~T} / \mathrm{m}$. The required quadrupole gradients of $250 \mathrm{~T} / \mathrm{m}$ can be achieved by using permanent magnet technology. Furthermore, our hybrid design of the CBII magnet takes advantage of the combination of smaller radial shifts of quadrupole poles installed in the wide gap of a long dipole magnet that produces an additional magnetic field. We developed a conceptual mechanical design of the complex bend II and calculated and analyzed the corresponding magnetic fields.

A prototype of the complex bend is being developed for magnetic measurements and will test with a beam at BNL's ATF at $50 \mathrm{MeV}$. In this paper, we developed and presented the scaling laws for the prototype.

\section{ACKNOWLEDGMENTS}

This research used resources of the National Synchrotron Light Source II, a U.S. Department of Energy (DOE) Office of Science User Facility operated for the DOE Office of Science by Brookhaven National Laboratory under Contract No. DE-SC0012704.

[1] T. Shaftan, V. Smaluk, and G. Wang, The concept of complex bend, Report No. BNL-211211-2019-TECH, https://technotes.bnl.gov/PDF?publicationId=211211.

[2] G. Wang, T. Shaftan, V. Smaluk, N. A. Mezentsev, S. Sharma, O. Chubar, Y. Hidaka, and C. Spataro, Complex bend: Strong-focusing magnet for low-emittance synchrotrons, Phys. Rev. Accel. Beams 21, 100703 (2018).

[3] T. Shaftan, G. Wang, V. Smaluk, Y. Hidaka, O. Chubar, T. Tanabe, and J. Choi, Complex bend II, Report No. BNL-211223-2019-TECH, https://technotes.bnl.gov/PDF? publicationId $=211223$.

[4] J. A. Uythoven, J. I. M. Botman, and H. L. Hagedoorn, Analytical evaluation of synchrotron radiation integrals, in Proceedings of 1st European Particle Accelerator Conference, Rome, Itlay, 1988, pp. 649-650, http:// accelconf.web.cern.ch/accelconf/e88/PDF/ EPAC1988_0649.PDF.

[5] S. Y. Lee, Accelerator Physics, 3rd ed. (World Scientific, Singapore, 2012).

[6] M. Borland, Elegant: A flexible SDDS-compliant code for accelerator simulation, Advanced Photon Source LS-287, 2000, https://www.aps.anl.gov/files/APS-sync/ 1snotes/files/APS_1418218.pdf.

[7] O. Chubar, P. Elleaume, and J. Chavanne, A 3D magnetostatics computer code for insertion devices, J. Synchrotron Radiat. 5, 481 (1998).

[8] O. Chubar and P. Elleaume, Accurate and efficient computation of synchrotron radiation in the near field region, in Proceedings of 6th European Particle Accelerator Conference, Stockholm, 1998, pp. 1177-1179, http://accelconf.web.cern.ch/Accelconf/e98/PAPERS/ THP01G.PDF.

[9] S. Sharma, N. A. Mezentsev, T. Shaftan, V. Smaluk, C. Spataro, T. Tanabe, and G. Wang, High gradient quadrpoles for low emittance synchrotrons, in Proceedings of 10th International Particle Accelerator Conference, Melbourne, Australia, 2019, pp. 4332-4334, http:// accelconf.web.cern.ch/AccelConf/ipac2019/papers/thpts094 .pdf.

[10] N. Tsoupas et al. Main magnets and correctors for the CBETA and eRHIC projects and hadron facilities, Phys. Procedia 90, 143 (2017). 